九州大学学術情報リポジトリ

Kyushu University Institutional Repository

\title{
Tests due to Pooling Data Through Preliminary Test on Biological Direct Assay
}

Asano, Choichiro

Shionogi Pharamaceutical Co. I Kyushu University

https://doi.org/10.5109/12992

出版情報 : 統計数理研究. 9 (2/3)，pp. 25-39，1960-10. Research Association of Statistical Sciences バージョン：

権利関係 : 


\title{
TESTS DUE TO POOLING DATA THROUGH PRELIMINARY TEST ON BIOLOGICAL DIRECT ASSAY
}

\author{
By \\ Chooichiro ASANO \\ (Received February 8, 1960)
}

\section{$\S 1$. Summary}

This paper discusses some methods for test of a mean effect on biological direct assay on the basis of pooling of data. A need for such methods is emphasized in the experiments under a restriction of small sample size at two or several similar environments or conditions, especially on biological direct assay. The principle of pooling of data is based on a scheme of $\mathrm{T}$. Kitagawa [1], B. E. B. Bennett [1] which discussed the estimation of a mean effect after preliminary test of significance. And recently $\mathrm{H}$. Bozivich, T. A. Bancroft and H. D. Hartley [1] discussed the power of analysis of variance test according to the pooling scheme of data. In this paper the tests of means and percentages after the preliminary test of significance are discussed and a recommendation is given from the viewpoints of the size of the power and the power gain.

\section{$\S 2$. Introduction}

The principle of a direct assay is that the doses of a standard preparation and those of a test preparation, both sufficient to produce a specified responce are directly measured and a difference of their potency can be investigated by their respective critical doses. Of course the simultaneous trial method is the basis of this assay techniques, and the test preparations $T$ can be assayed relative to a standard preparation $S$ by simultaneous experimentation by the comparison between the observed doses of $S$ and the observed doses of $T$ under the same environments and conditions.

The fundamental idea of this paper is that if such a standard pattern of assay has been adopted as a routine in factory production or elsewhere, a pooling of data can be introduced, in order to reinforce the power of test under a restriction of numbers of subjects. In some circumstances we had better pool the data in repeated assay of $S$, and it is the purpose of this paper to formulate such a rule of pooling procedure and to clarify the

\begin{tabular}{c|cc}
\hline Preparation & Control & Test \\
\hline Block(Days) & & \\
\hline 1 & $S_{(1)}$ & $T_{1}$ \\
2 & $S_{(2)}$ & $T_{2}$ \\
\hline
\end{tabular}
consequences of the pooling procedure.

When a direct assay has performed as the following (left-sided) figure, a rule of test procedure for $T_{2}$ naturally may take the following steps: 
(i) Make a statistical test on a distinction (mean or percentage) between $S_{(1)}$ and $S_{(2)}$ with a significant level $\alpha_{1}$.

(ii) Then

(a) if the significance of the difference between $S_{(1)}$ and $S_{(2)}$ is recognized, a similar statistical test between $S_{(2)}$ and $T_{2}$ is made only in block 2 with a significant level $\alpha_{2}$,

(b) otherwise, that is, if the significance of the difference is not recognized, the data of $S_{(1)}$ and $S_{(2)}$ are pooled and a similar test between $S_{(1)+(2)}$ and $T_{2}$ is made with a significant level $\alpha_{3}$, where $S_{(1)+(2)}$ means the pooled data of $S_{(1)}$ and $S_{(:)}$.

In $\S 3$ we discuss a test on normal distribution having a common krown variance, which at the same time gives us a test of percentage by means of usual normal approximation, and in $\S 4$. we apply a $t$-test procedure between $S$ and $T_{2}$, where two normal universes are assumed to have a common, but unknown variance. $\$ 5$ discusses a test of percentage by means of double dichotomies. In $\S 3$ and $\S 5$, some numerical considerations are given.

The author wishes his hearty thanks to Prof. T. Kitagawa for his kind suggestions and encouragement.

\section{$\S 3$. $u$-test after preliminary $u$-test of significance}

Let $O_{N_{1}}:\left(x_{11}, x_{12}, \cdots, x_{1 v_{1}}\right)$ be a random sample of $N_{1}$ from a normal universe $N\left(\mu_{1}, \sigma^{2}\right)$, which indicates the critical doses observed on $S$ in block 1 , and $O_{N_{2}}:\left(x_{21}, x_{22}, \cdots, x_{2 v_{2}}\right)$ be a random sample of $N_{2}$ from other normal universe $N\left(\mu_{2}, \sigma^{2}\right)$, which indicates the critical doses observed on $S$ in block 2 , and then let $O_{N_{3}}:\left(x_{31}, x_{32}, \cdots, x_{3 N_{3}}\right)$ be a random sample of $N_{3}$ from the third normal universe $N\left(\mu_{3}, \sigma^{2}\right)$, which indicates the critical doses observed on $T_{2}$ in block 2. These three normal universes are assumed to have a common universe variance, whose value is known to us. The difference between $S_{(1)}$ and $S_{(2)}$ may be regarded to be due to a fluctuation on blocks, which may exist or may not.

Let us suppose that we shall pool two groups of sample observations on $S_{(1)}$ and $S_{(2)}$ when the hypothesis $\mu_{1}=\mu_{2}$ cannot be rejected and then let us test the difference between $T_{2}$ and $S$ by the pooled observations.

Our rule of test procedure is formulated as follows:

(1) Let a statistic $u_{1}$ be defined by

$$
u_{1}=\left(\bar{x}_{1}-\bar{x}_{2}\right) / \sqrt{\frac{1}{N_{1}}+\frac{1}{N_{2}}} \sigma \text {, }
$$

where $\bar{x}_{i}=\sum_{j=1}^{N i} x_{i j} / N_{i}$. Let $u\left(\alpha_{i}\right)$ be the $\alpha_{i}$-point of the normal distribution $N(0,1)$ such that 


$$
(\sqrt{2 \pi})^{-1} \int_{-u\left(\alpha_{i}\right)}^{u\left(\alpha_{i}\right)} e^{-\frac{t^{2}}{2}} d t=-\alpha_{i}, \quad(i=1,2,3) .
$$

(2) Let us introduce a test for $T_{2}$ defined in the following manner.

(a) If $u_{1} \geq u\left(\alpha_{1}\right)$, then test the hypothesis $H: \mu_{2}=\mu_{3}$ by applying the normal test to the statistic

$$
u_{2}=\left(\bar{x}_{2}-x_{3}\right) / \sqrt{\frac{1}{N_{2}}+\frac{1}{N_{3}}} \sigma
$$

with the significance level 'a.

(b) Otherwise, that is, if $\boldsymbol{u}_{1} \mid<u\left(\alpha_{1}\right)$, then assuming $\mu_{1}=\mu_{2}=\mu_{12}$, say, test the hypothesis $H: \mu_{12}=\mu_{3}$ by applying the normal test to the statistic

$$
u_{3}=\left(\bar{x}_{12}-\bar{x}_{3}\right) / \sqrt{\frac{1}{N_{12}}+\frac{1}{N_{3}}} \sigma,
$$

with the significance level $\alpha_{3}$, where $\bar{x}_{12}=\left(N_{1} \bar{x}_{1}+N_{2} \bar{x}_{2}\right) / N_{12}$ and $N_{12}=N_{1}+$ $N_{2}$.

Then the power of our test procedure are of importance in this problem, and in consequence of these considerations the values of $\alpha_{1}, \alpha_{2}$ and $\alpha_{3}$ are proposed for the practical uses so as to keep approximately an assigned value for the power of our test.

The power of our test procedure is given by

$$
\operatorname{Pr} .\left\{D_{1}\right\}+\operatorname{Pr} .\left\{D_{2}\right\}=\iint_{D_{1}} f_{1}\left(y_{1}, y_{2}\right) d y_{1} d y_{2}+\iint_{\nu_{2}} f_{2}\left(y_{1}, y_{3}\right) d y_{1} d y_{3},
$$

where we put

$$
\begin{gathered}
f_{1}\left(\boldsymbol{y}_{1}, \boldsymbol{y}_{2}\right)=\left(2 \pi \sigma_{1} \sigma_{2 V} 1-\rho_{1}^{2}\right)^{-1} \exp \left[-\frac{1}{2\left(1-\rho_{1}^{2}\right)}\left\{\frac{\left(y_{1}-\eta_{1}\right)^{2}}{\sigma_{1}^{2}}-\frac{2 \rho_{1}\left(y_{1}-\eta_{1}\right)\left(y_{2}-\eta_{2}\right)}{\sigma_{1} \sigma_{2}}\right.\right. \\
\left.\left.+\frac{\left(y_{2}-\eta_{2}\right)^{2}}{\sigma_{2}^{2}}\right\}\right],
\end{gathered}
$$

$$
\left(f_{2}\left(y_{1}, y_{3}\right)=\left(2 \pi \sigma_{1} \sigma_{2}\right)^{-1} \exp \left[-\frac{1}{2}\left\{\frac{\left(y_{1}-\eta_{1}\right)^{2}}{\sigma_{1}^{2}}+\frac{\left(y_{3}-\eta_{3}\right)^{2}}{\sigma_{3}^{2}}\right\}\right],\right.
$$

$$
\left\{\begin{array}{l}
y_{1}=x_{1}-\bar{x}_{2}, y_{2}=\bar{x}_{2}-\bar{x}_{3}, \quad y_{3}=\frac{N_{1}}{N_{1}+N_{2}} \bar{x}_{1}+\frac{N_{2}}{N_{1}+N_{2}} \bar{x}_{2}-\bar{x}_{3}, \\
\sigma_{1}=\sigma \sqrt{\frac{1}{N_{1}}+\frac{1}{N_{2}}}, \quad \sigma_{2}=\sigma \sqrt{\frac{1}{N_{2}}+\frac{1}{N_{3}}}, \sigma_{3}=\sigma \sqrt{\frac{1}{N_{1}+N_{2}}+\frac{1}{N_{2}}} \\
\eta_{1}=\mu_{1}-\mu_{2}, \eta_{2}=\mu_{2}-\mu_{3}, \quad \eta_{3}=\frac{N_{1}}{N_{1}+N_{2}} \mu_{1}+\frac{N_{2}}{N_{1}+N_{2}} \mu_{2}-\mu_{3},
\end{array}\right.
$$




$$
\left.\rho_{1}=-\left\{N_{2} \sqrt{\left(\frac{1}{N_{1}}+\frac{1}{N_{2}}\right)}\right)\left(\frac{1}{N_{2}}+\frac{1}{N_{3}}\right)\right\}^{-1}, \quad \rho_{2}=0
$$

and

$$
\begin{aligned}
& D_{1}: \quad \sigma \sqrt{\frac{1}{N_{1}}+\frac{1}{N_{2}}} u\left(\alpha_{1}\right) \leq y_{1}<\infty, \\
& \sigma \sqrt{\frac{1}{N_{2}}+\frac{1}{N_{3}}} u\left(\alpha_{2}\right) \leq y_{2}<\infty, \\
& D_{2}:\left(\begin{array}{l}
0<y_{1}<\sigma \sqrt{\frac{1}{N_{1}}+\frac{1}{N_{2}}} u\left(\alpha_{1}\right), \\
\sigma \sqrt{\frac{1}{N_{12}}+\frac{1}{N_{3}}} u\left(\alpha_{3}\right)<y_{3}<\infty .
\end{array}\right.
\end{aligned}
$$

For the test of percentages, a condition of some normal approximation may be more or less satisfied, and as the simple normal approximation the observations $p_{1}=\left(\boldsymbol{r}_{1} / N_{1}\right), p_{2}=\left(\boldsymbol{r}_{2} / N_{2}\right)$ and $p_{3}=\left(\boldsymbol{r}_{3} / N_{3}\right)$ can be considered by arcsine transformation, $\sin ^{-1}, \bar{r}_{i} / N_{i},(i=1,2,3)$, and then their variances are known to be approximately the constants, that is, $\left(4 N_{i}\right)^{-1}$. In this sicuation the above results for test on normal distributions with known variance are directly applicable for that on percentages.

Now we attempt to give numerical informations about the size of power and the power gains by numerical evaluations of (3.4) by assigning some values of the pairs of $\left\{N_{i}, \alpha_{i}\right\},(i=1,2,3)$. The power gains are used to compare our sometimes pool procedure with that of the neverpool test and are defined as follows:

(i) Assume a value of the parameter $\eta_{1}$ to be zero:

(ii) For this value of $\eta_{1}$, evaluate the size of the sometimes-pool test.

(iii) For this level of size, evaluate the power curve of the never-pool test: this power curve is then directly comparable with that of the sometimes-pool test corresponding to $\eta_{1}=0$.

The behaviour of the size of the power and the power gain are illustrated as a function of $\left(\eta_{1} / \sigma, \eta_{2} / \sigma\right)$ for certain values of $N_{1}, N_{2}, N_{3}$ and $\alpha_{1}$, $\alpha_{2}, \alpha_{3}$ : Fig. $1\left(N_{1}=N_{2}=N_{3}=4, \alpha_{1}=\alpha_{2}=\alpha_{3}=0.05\right)$, Fig. $2\left(N_{1}=N_{2}=N_{3}=4\right.$, $\left.\alpha_{1}=0.2, \alpha_{2}=\alpha_{3}=0.05\right)$, Fig. $3\left(N_{1}=N_{2}=N_{3}=4, \alpha_{1}=\alpha_{2}=\alpha_{3}=0.10\right)$; Fig. 4 $\left(N_{1}=N_{2}=N_{3}=10, \alpha_{1}=\alpha_{2}=\alpha_{3}=0.10\right)$.

So far as these numerical data are concerned, we can make the following observations which might be suggestive to our statistical procedure in more general situation.

(i) The size of our test procedure does not equal to the nominal level of 0.05 (or 0.10 ), but varies as $\alpha_{1}$ and $\eta_{2} / \sigma$ vary. It can be noted that the size of the power for the values of $\eta_{2} / \sigma$ between 0 and 2 in the case of $\left(\eta_{1}=0\right.$, and $\left.\alpha_{2}=\alpha_{3}=0.05\right)$ is larger than 0.05. As $\eta_{2} / \sigma$ becomes larger, the size peak tends to the left and becomes larger than the nominal pool test. 
$\left(A_{1}\right)$

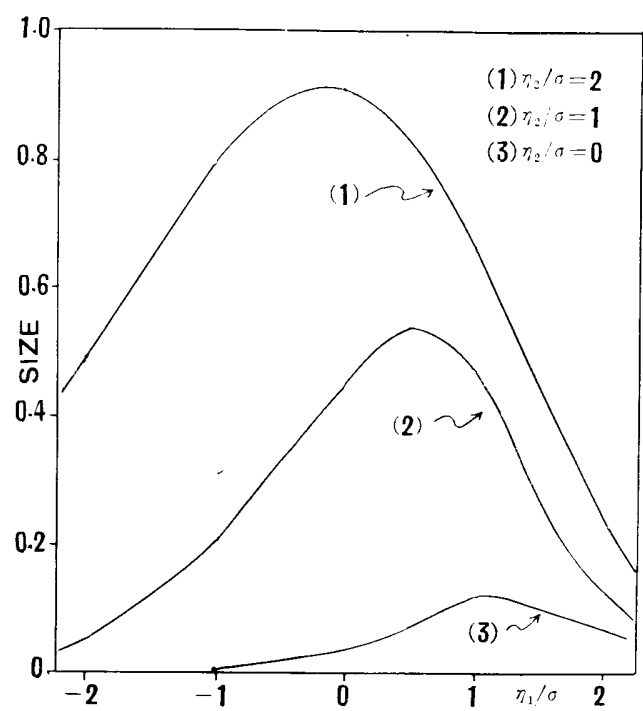

Fig. 1. (a) Size curves for $N_{1}=N_{2}=N_{3}=4$, $\alpha_{1}=\alpha_{2}=\alpha_{3}=0.05$

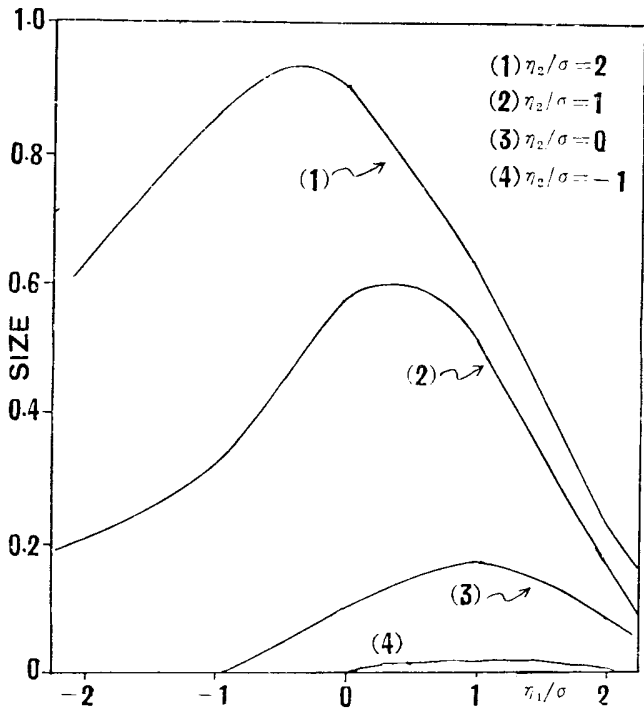

Fig. 3. (a) Size curves for $N_{1}=N_{2}=N_{3}=4$, $\alpha_{1}=\alpha_{2}=\alpha_{3}=0.10$

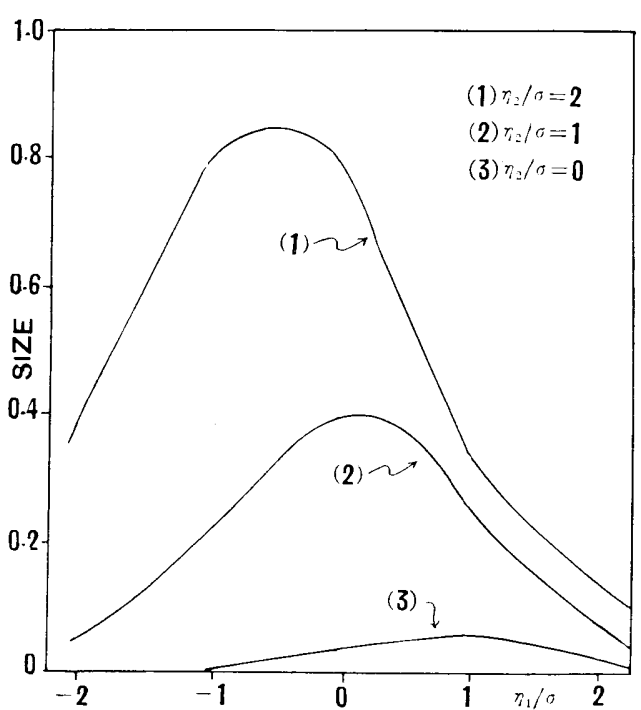

Fig. 2. (a) Size curves for $N_{1}=N_{2}=N_{3}=4$, $\alpha_{1}=0.2, \alpha_{2}=\alpha_{3}=0.05$

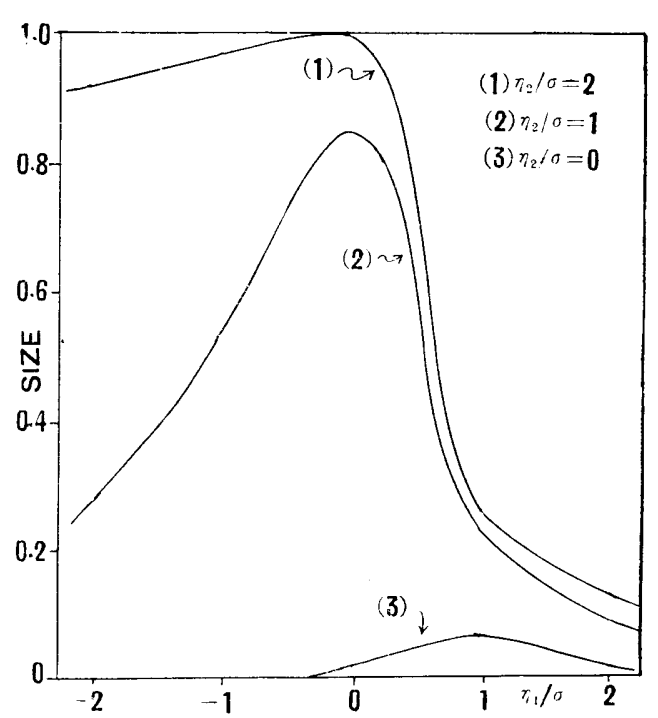

Fig. 4. (a) Size curves for $N_{1}=N_{2}=N_{3}=10$, $\alpha_{1}=\alpha_{2}=\alpha_{3}=0.10$ 
$\left(A_{2}\right)$

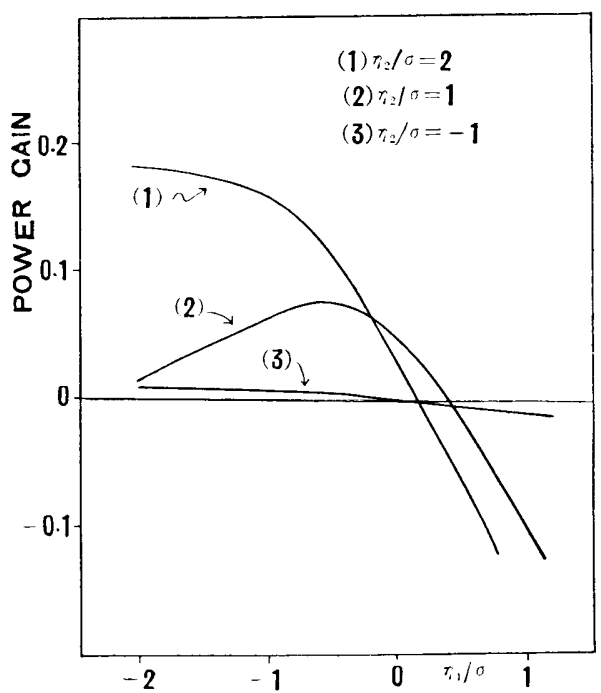

Fig. 1. (b) Power gain of the sometimes-pool procedure over teh never-pool test of the same size for $N_{1}=N_{2}=N_{3}=4, \alpha_{1}=\alpha_{2}=\alpha_{3}=0.05$

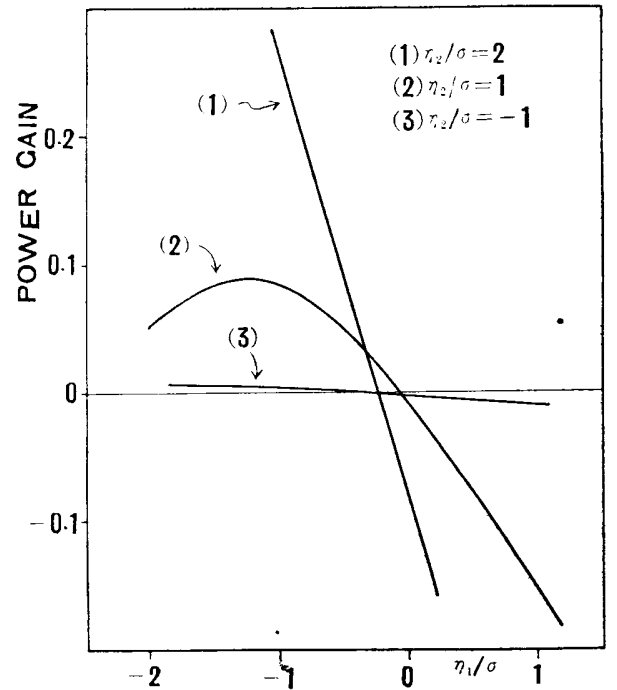

Fig. 2. (b) Power gain for $N_{1}=N_{2}=\Lambda_{3}$ $=4, \alpha_{1}=0.2, \alpha_{2}=\alpha_{3}=0.05$

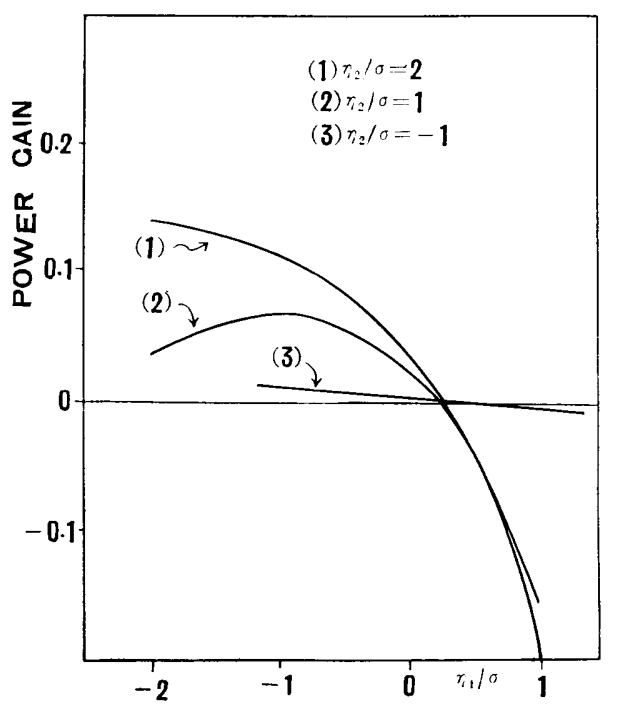

Fig. 3. (b) Power gain for $N_{1}=N_{2}=N_{3}=4$, $\alpha_{1}=\alpha_{2}=\alpha_{3}=0.10$

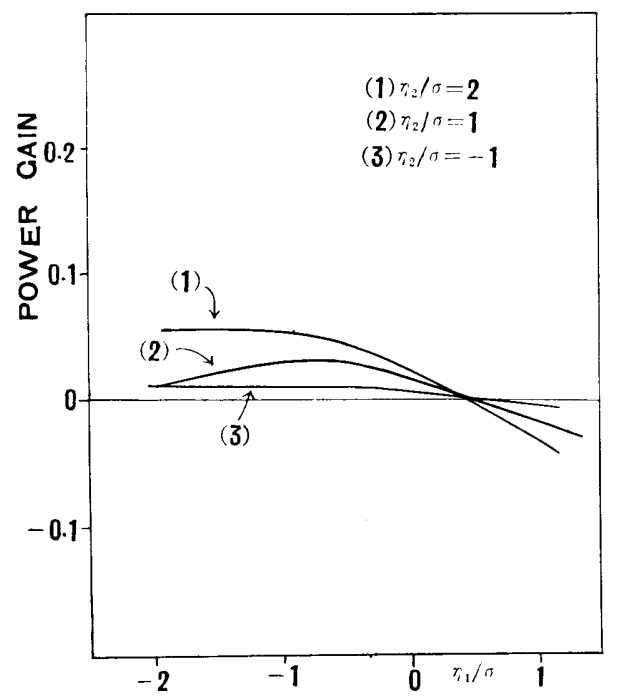

Fig. 4. (b) Power gain for $N_{1}=N_{2}=N_{3}=10$, $\alpha_{1}=\alpha_{2}=\alpha_{3}=0.10$ 
Moreover, the larger $\eta_{2} / \sigma$ becomes, the narrower becomes the interval in which the size of the power exceeds that of the nominal pool test.

(ii) Referring to the size curve for a preliminary test carried out at the 0.05 level, the peak is usually high. Clearly, a preliminary test carried out at this level will in many cases admit an unacceptable size disturbance. Therefore, with the intention of size control for the nominal level of 0.05 , that is, in order to decrease the peak of the size or to narrow the interval which exceeds the power of the never-pool test, we may propose a statistical procedure taking 0.20 as the value of $\alpha_{1}$ with $\alpha_{2}=\alpha_{3}=0.05$.

(iii) From the point of the power gain, if we can assume that $0.5 \geq \eta_{1} / \sigma$ $\geq-2$, we may find a large power gain by choosing the level $\alpha_{1}=0.20$. However, under the assumption $0.5<\eta_{1} / \sigma$, the power gain is less than the never-pool test. And if we have nothing to assume, we may propose a borderline test.

(iv) The larger $N\left(=N_{i}\right)$ becomes, the smaller the power gain is obtained.

\section{\$4. $t$-test after preliminary $t$-test of significance}

Let $O_{N_{1}}:\left(x_{11}, x_{12}, \cdots, x_{1 v_{1}}\right), O_{N_{2}}:\left(x_{21}, x_{22}, \quad, x_{2 v_{2}}\right)$ and $O_{N_{3}}:\left(x_{31}, x_{32}, \cdots\right.$, $\left.x_{3 v_{3}}\right)$ be random sample from normal universe $N\left(\mu_{1}, \sigma_{2}\right), N\left(\mu_{2}, \sigma^{2}\right)$ and $N\left(\mu_{3}\right.$, $\left.\sigma^{2}\right)$, respectively, with the same meaning as in $\S 3$. In this section, the three normal universes are assumed to have a common but unknown variance $\sigma^{2}$. The universe means $\mu_{1}, \mu_{2}$ and $\mu_{3}$ are not necessarily assumed to be equal.

In this case, our rule of test procedure is defined as follows.

(i) Let $\bar{x}_{1}, \bar{x}_{2}$ and $\bar{x}_{3}$ be sample means and let $s_{1}^{2}, s_{2}^{2}$ and $s_{3}^{2}$ be sample variances respectively.

(ii) Let a statistic $t_{1}$ be defined by

$$
\left.t_{1}=\left(\bar{x}_{1}-\bar{x}_{2}\right) /\left(\frac{1}{N_{1}}+\frac{1}{N_{2}}\right) \cdot \frac{\left(N_{1}-1\right) s_{1}^{2}+\left(N_{2}-1\right) s_{2}^{2}}{N_{1}+N_{2}-2}\right)^{-\frac{1}{2}},
$$

where $\bar{x}_{i}=\sum_{j=1}^{N i} x_{i j} / N_{i}, \quad s_{i}^{2}=\sum_{j=1}^{N i}\left(x_{i j}-\bar{x}_{i .}\right)^{2} /\left(N_{i}-1\right)$,

and let $t_{\phi}\left(\alpha_{i}\right)$ be a significance value of $t$-distribution with the $\phi$ degrees of freedom and the significance level $\alpha_{i},(i=1,2,3)$.

(iii) Let us introduce a test for $T_{2}$ defined in the following manner: (a) If $t_{1}>t_{N_{1}+N_{2}-2}\left(\alpha_{1}\right)$, then test the hypothesis $H: \mu_{2}=\mu_{3}$ by applying the t-test to the statistic

$$
t_{2}=\left(\bar{x}_{2}-\bar{x}_{3}\right)\left(\frac{1}{N_{2}}+\frac{1}{N_{3}}\right)^{\frac{1}{2}}\left(\frac{\left(N_{2}-1\right) s_{1}^{2}+\left(N_{3}-1\right) s_{3}^{2}}{N_{2}+N_{3}-2}\right)^{\frac{1}{2}},
$$

with the significance level $\alpha_{2}$.

(b) Otherwise, that is, if $t_{1}^{\prime} \leq t_{N_{1}+x_{2}-!}\left(\alpha_{1}\right)$, then assuming $\mu_{1}=\mu_{2}$ $=\mu_{12}$, say, test the hypothesis $H: \mu_{12}=\mu_{3}$ by applying the t-test to the 
statistic

$$
t_{3}=\left(\bar{x}_{12}-\bar{x}_{3}\right)\left(\frac{1}{N_{12}}+\frac{1}{N_{3}}\right)^{\frac{1}{2}}\left(\frac{\left(N_{12}-2\right) s_{12}^{2}+\left(N_{3}-1\right) s_{3}^{2}}{N_{12}+N_{3}-2}\right)^{\frac{1}{2}}
$$

with the significance level $\alpha_{3}$, where

$$
\begin{aligned}
& \bar{x}_{12}=\left(N_{1} \bar{x}_{1}+N_{2} \bar{x}_{2}\right) /\left(N_{1}+N_{2}\right), \\
& s_{12}^{2}=\left\{\left(N_{1}-1\right) s_{1}^{2}+\left(N_{2}-1\right) s_{2}^{2}\right\} /\left(N_{1}+N_{2}-2\right) .
\end{aligned}
$$

Now our problem is to obtain the power of the test given by the above test procedure, as in $\S 3$, and this problem can be reduced the determination of the value of $\alpha_{1}, \alpha_{2}$ and $\alpha_{3}$ for the practical uses so as to keep approximately an assigned value of the power of test.

The power is denoted as follows.

$$
\begin{aligned}
\operatorname{Pr} .\left\{D_{1}\right\}+\operatorname{Pr} .\left\{D_{2}\right\}= & \operatorname{Pr} .\left\{\left|t_{1}\right|>t_{N_{1}+N_{2}-2}\left(\alpha_{1}\right), \mid t_{2}>t_{N_{2}+N_{3}-2}\left(\alpha_{2}\right)\right\} \\
& +\operatorname{Pr} .\left\{t_{1} \leq t_{N_{1}+N_{2}-2}\left(\alpha_{1}\right), t_{3}>t_{N_{1}+N_{3}-2}\left(\alpha_{3}\right)\right\} .
\end{aligned}
$$

Now let us put, for the moment,

$$
\begin{aligned}
& y_{1}=\bar{x}_{1}-\bar{x}_{2}, \quad y_{2}=\bar{x}_{2}-\bar{x}_{3}, \\
& n_{i}=N_{i}-1
\end{aligned}
$$

and

$$
W_{1}=-\frac{n_{1} s_{1}^{2}+n_{2} s_{2}^{2}}{\sigma^{2}}, \quad W_{2}=-\frac{n_{2} s_{2}^{2}+n_{3} s_{3}^{2}}{\sigma^{2}}, \quad W_{3}=\frac{n_{3} s_{3}^{2}}{\sigma^{2}} .
$$

Then the joint elementary probability of $\left(W_{1}, W_{2}, W_{3}\right)$ is shown by (4.8)

$$
\begin{gathered}
G\left(W_{1}, W_{2}, W_{3}\right) d W_{1} d W_{2} d W_{3} \\
=\frac{2^{-\left(n_{1}+n_{2}+n_{3}\right) / 2}}{\Gamma\left(\frac{n_{1}}{2}\right) \Gamma\left(\frac{n_{2}}{2}\right) \Gamma\left(\frac{n_{3}}{2}\right)}\left(W_{1}-W_{3}\right)^{\frac{n_{1}}{2}-1}\left(W_{2}-W_{3}\right)^{\frac{n_{2}}{2}-1} W_{3}^{\frac{n_{3}}{2}-1} e^{-\frac{W_{1}+W_{2}-W_{3}}{2}} \\
\cdot d W_{1} d W_{2} d W_{3}
\end{gathered}
$$

and we obtain the probability of $\left(W_{1}, W_{2}\right)$, integrating out $W_{3}$, as follows.

$$
\begin{aligned}
& +\operatorname{Pr} .\left\{W_{1} \leq W_{2}\right\}\left[\int_{0}^{W_{1}} G\left(W_{1}, W_{2}, W_{3}\right) d W_{3}\right] d W_{2} d W_{1}
\end{aligned}
$$

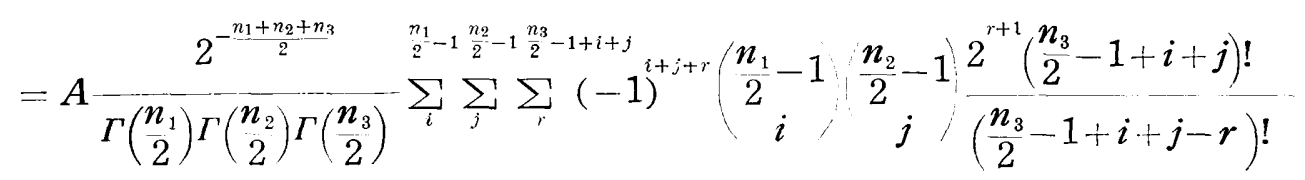


$\cdot W_{1}^{\frac{n_{1}}{2}-1-i} W_{2}^{\frac{n_{2}+n_{3}}{2}-2+i-r} e^{-\frac{W_{1}}{2}} d W_{2} d W_{1}+(1-A) \frac{2^{-\frac{n_{1}+\frac{n_{2}}{2}+n_{3}}{2}}}{\Gamma\left(\frac{n_{1}}{2}\right) \Gamma\left(\frac{\boldsymbol{n}_{2}}{2}\right) \Gamma\left(\frac{\boldsymbol{n}_{3}}{2}\right)} \sum_{i} \sum_{j} \sum_{r}(-1)^{i+j+r}$

$\cdot\left(\begin{array}{c}\boldsymbol{n}_{1} \\ 2 \\ i\end{array}\left(\begin{array}{c}\frac{\boldsymbol{n}_{2}}{2}-1 \\ j\end{array}\right) \frac{2^{r+1}\left(\frac{\boldsymbol{n}_{3}}{2}-1+i+j\right) !}{\left(\frac{\boldsymbol{n}_{3}}{2}-1+i+j-r\right) !} W_{1}^{\frac{n_{1}+n_{3}}{2}-2+j-1} W_{2}^{\frac{n_{0}}{2}+1-j} e^{-\frac{W_{3}}{2}} d W_{2} d W_{1}\right.$,

where

$$
A=\operatorname{Pr} \cdot\left\{\frac{n_{1} s_{1}^{2}}{\sigma^{2}} / \frac{n_{3} s_{3}^{2}}{\sigma^{2}}>1\right\}=\int_{n_{3} / n_{1}}^{\infty} h_{n_{1}, n_{3}}(F) d F .
$$

But the joint probability of $\left(y_{1}, y_{2}\right)$ is shown by

$$
\begin{aligned}
f\left(y_{1}, y_{2}\right) d y_{1} d y_{2}= & \left(2 \pi \sigma_{1} \sigma_{2} \gamma^{\prime} 1-\rho^{2}\right)^{-1} \exp \left[-\frac{1}{2\left(1-\rho^{2}\right)}\right. \\
& \left.\cdot\left\{\frac{\left(y_{1}-\eta_{1}\right)^{2}}{\sigma_{1}^{2}}-\frac{2 \rho\left(y_{1}-\eta_{1}\right)\left(y_{2}-\eta_{2}\right)}{\sigma_{1} \sigma_{2}}+\frac{\left(y_{2}-\eta_{2}\right)^{2}}{\sigma_{2}^{2}}\right\}\right] d y_{1} d y_{2},
\end{aligned}
$$

where

$$
\begin{aligned}
& \sigma_{1}=\sigma \sqrt{\frac{1}{N_{1}}+\frac{1}{N_{2}}}, \quad \sigma_{2}=\sigma / \frac{\overline{1}}{\overline{N_{2}}+\frac{1}{N_{3}}}, \eta_{1}=\mu_{1}-\mu_{2}, \eta_{2}=\mu_{2}-\mu_{3} \text {, } \\
& \rho=-\left\{n_{2} \sqrt{\left(\frac{1}{N_{1}}+\frac{1}{N_{2}}\right)\left(\frac{1}{N_{2}}+\frac{1}{N_{3}^{-}}\right)}\right\}^{-1}
\end{aligned}
$$

Consequently, the joint probability of $\left(W_{1}, W_{2}, y_{1}, y_{2}\right)$ is obtained by

$$
H\left(W_{1}, W_{2}\right) f\left(y_{1}, y_{2}\right) d W_{1} d W_{2} d y_{1} d y_{2},
$$

and further let us transform $\left(W_{1}, W_{2}, y_{1}, y_{2}\right)$ into $\left(T_{1}, T_{2}\right)$ by

$$
T_{1}=y_{1} / \checkmark \overline{W_{1}} \sigma_{1}, T_{2}=y_{2} / \sqrt{W_{2}} \sigma_{2} .
$$

As a result, throughout the troublesome calculations, the first term of the power is expressed by the following function,

$$
\begin{gathered}
\operatorname{Pr} .\left\{D_{1}\right\}=A \frac{\exp \left\{\frac{-1}{2\left(1-\rho^{2}\right)}\left(\frac{\eta_{1}^{2}}{\sigma_{1}^{2}}-2 \frac{\rho}{\sigma_{1} \eta_{2}}+\frac{\eta_{2}^{2}}{\sigma_{2}}+\frac{\boldsymbol{n}_{2}^{2}}{\sigma_{2}^{2}}\right)\right\}}{\pi \Gamma\left(\frac{\boldsymbol{n}_{1}}{2}\right) \Gamma\left(\frac{\boldsymbol{n}_{2}}{2}\right) \Gamma\left(\frac{\boldsymbol{n}_{3}}{2}\right)} \sum_{i=0}^{\frac{n_{1}}{2} \sum_{j=0}^{\frac{n_{2}}{2}-1} \sum_{r=0}^{\frac{n_{2}}{2}-1+i+j}(-1)^{i+j+r}} \\
\cdot\left(\begin{array}{c}
\frac{\boldsymbol{n}_{1}}{2}-1 \\
i
\end{array}\right)\left(\begin{array}{c}
\frac{\boldsymbol{n}_{2}}{2}-1 \\
j
\end{array}\right)\left(\begin{array}{c}
\frac{\boldsymbol{n}_{3}}{2}-1+\boldsymbol{i}+\boldsymbol{j} \\
\boldsymbol{r}
\end{array}\right) r ! \sum_{a=0}^{\infty} \sum_{b=0}^{\infty} \sum_{c=0}^{\infty} 2^{\frac{a+b+2 c}{2}}\left(1-\rho^{2}\right)^{\frac{n_{1}+n_{2}+n_{3}}{2}-\frac{\alpha+b}{2}-r-\frac{1}{2}}
\end{gathered}
$$




$$
\begin{aligned}
& \cdot \frac{\left(\frac{\eta_{1}}{\sigma_{1}}-\rho \frac{\eta_{2}}{\sigma_{2}}\right)^{a}\left(\frac{\eta_{2}}{\sigma_{2}}-\rho \frac{\eta_{1}}{\sigma_{1}}\right)^{b} \rho^{a}}{a ! b ! c !}\left\{\Gamma\left(\frac{n_{1}}{2}-i-\frac{a+c+1}{2}\right) \Gamma\left(\frac{n_{2}+n_{3}}{2}+i-r+\frac{b+c-1}{2}\right)\right. \\
& \cdot \iint_{D_{1}}\left(\frac{1}{T_{2}^{2}}\right)^{\frac{n_{2}+n_{3}}{2}+i-r-\frac{1}{2}}\left(\frac{1}{T_{1}^{2}}\right)^{\frac{n_{1}}{2}-i+\frac{1}{2}}\left(\frac{T_{1}^{2}}{\left(1-\rho^{2}\right)+T_{1}^{2}}\right) d T_{1} d T_{2}+\Gamma\left(\frac{n_{1}+n_{3}}{2}+j-r+\frac{a+c-1}{2}\right) \\
& \left.\cdot \Gamma\left(\frac{n_{2}-i+c+c+1}{2}-j+\frac{b+c+1}{2}\right) \iint_{D_{1}}\left(\frac{1}{T_{1}^{2}}\right)^{\frac{n_{1}+n_{3}}{2}+j-r-\frac{1}{2}} \quad\left(\frac{1}{T_{2}^{2}}\right)^{\frac{n_{2}}{2}-j+\frac{1}{2}} \quad\left(\frac{T_{2}^{2}}{\left(1-\rho^{2}\right)+T_{2}^{2}}\right) d T_{1} d T_{2}\right\},
\end{aligned}
$$

where $D_{1}$ shows the domain of $\left(T_{1}, T_{2}\right)$ such that

$$
\frac{\sqrt{\boldsymbol{n}_{1} \boldsymbol{n}_{2}}}{\boldsymbol{n}_{1}+\boldsymbol{n}_{2}} \boldsymbol{n}_{N_{1}+N_{2}-2}\left(\alpha_{1}\right) \leq \boldsymbol{T}_{1}<\infty, \quad \frac{\sqrt{\boldsymbol{n}_{2} \boldsymbol{n}_{3}}}{\boldsymbol{n}_{2}+\boldsymbol{n}_{3}} t_{N_{2}+N_{3}-2}\left(\alpha_{2}\right) \leq T_{2}<\infty .
$$

Similarly the second term of the power is obtained from the joint distribution of $\left(W_{1} W_{3}, y_{1}, y_{3}\right)$, that is,

$$
\begin{aligned}
& H\left(W_{1}, W_{3}\right) f\left(y_{1}, y_{3}\right) d W_{1} d W_{3} d y_{1} d y_{3}=\frac{2^{\left.-\frac{n_{1}+n_{2}+n_{3}}{2}+1\right)}}{\pi \sigma_{1} \sigma_{3} \Gamma\left(\frac{n_{1}+n_{2}}{2}\right) \Gamma\left(\frac{n_{3}}{2}\right)}\left(W_{3}-W_{1}\right)^{\frac{n_{3}}{2-1}} \\
& \cdot W_{1}^{n_{1}+n_{2}-1} \exp \left[-\frac{1}{2}\left\{W_{3}+\frac{\left(y_{1}-\eta_{1}\right)^{2}}{\sigma_{1}^{2}}+\frac{\left(y_{3}-\eta_{3}\right)^{2}}{\sigma_{3}^{2}}\right\}\right] d W_{1} d W_{3} d y_{1} d y_{3},
\end{aligned}
$$

where we put

$$
\begin{aligned}
& y_{1}=\bar{x}_{1}-\bar{x}_{3}, \quad y_{3}=\frac{N_{1}}{N_{1}+N_{2}}-\bar{x}_{1}+\frac{N_{2}}{N_{1}+N_{2}} \bar{x}_{2}-\bar{x}_{3}, \\
& \sigma_{1}=\sqrt{\frac{1}{N_{1}}+\frac{1}{N_{2}}} \sigma, \sigma_{3}=\sqrt{\frac{1}{N_{2}+N_{3}}+\frac{1}{N_{1}}} \sigma, \rho_{2} \equiv \rho\left(y_{1}, y_{3}\right)=0, \\
& \eta_{1}=\mu_{1}-\mu_{2}, \quad \eta_{3}=\frac{N_{1}}{N_{1}+N_{2}} \mu_{1}+\frac{N_{2}}{N_{1}+N_{2}} \mu_{2}-\mu_{3} .
\end{aligned}
$$

Now let us transform $\left(W_{1}, W_{3}, y_{1}, y_{3}\right)$ into the following $\left(T_{1}, T_{3}\right)$

$$
T_{1}=y_{1} / \overline{W_{1}} \sigma_{1}, \quad T_{3}=y_{3} / \checkmark \overline{W_{3}} \sigma_{3},
$$

and then we obtain the following power function.

$$
\operatorname{Pr} .\left\{D_{2}\right\}=\frac{2^{-\left(\frac{n_{1}+n_{2}+n_{3}}{2}+1\right)}}{\pi \Gamma\left(\frac{n_{1}+n_{2}}{2}\right) \Gamma\left(\frac{n_{3}}{2}\right)} \sum_{i=0}^{\frac{n_{3}}{2}-1}(-1) \iint_{\nu_{2}}^{\frac{n_{1}+n_{2}-1}{2} W_{1}^{2}+i} W_{3}^{\frac{n_{3}-1}{2}-i}
$$




$$
\cdot \exp \left[-\frac{1}{2}\left\{W_{3}+\left(T_{1} W_{1}^{1 / 2}-\frac{\eta_{1}}{\sigma_{1}}\right)^{2}+\left(T_{3} W_{3}^{1 / 2}-\frac{\eta_{3}}{\sigma_{3}}\right)^{2}\right\}\right] d T_{1} d T_{3} d W_{1} d W_{3}
$$

where $D_{2}$ shows the interval

$$
\left\{\begin{array}{l}
0<T_{1}<\frac{\sqrt{\boldsymbol{n}_{1} \boldsymbol{n}_{2}}}{\boldsymbol{n}_{1}+\boldsymbol{n}_{2}} t_{N_{1}+N_{2}-2}\left(\alpha_{1}\right), \frac{\sqrt{\boldsymbol{n}_{3}\left(\boldsymbol{n}_{1}+\boldsymbol{n}_{2}\right)}}{\boldsymbol{n}_{1}+\boldsymbol{n}_{2}+\boldsymbol{n}_{3}} t_{N_{12}+N_{3}-2}\left(\alpha_{3}\right) \leq T_{3}<\infty . \\
0<W_{1}<\infty, 0<W_{3}<\infty .
\end{array}\right.
$$

The power defined by the formula (4.5) can now be written in terms of the right hand-sides of (4.15) and (4.20), and a paper of the author is being prepared which will give the numerical tables with various $\left(\eta_{1} / \sigma_{1}\right)$, $\left(\eta_{2} / \sigma_{2}\right)$ and $N_{1}, N_{2}, N_{3}$ using magnetic drum data-processing machine, where we may apply the Monte Carlo method with some considerations to the formula (4.5).

\section{Other test for percentages after preliminary test for percentages}

In simultaneous trials, whether they may be measured by attributes or not, a method of double dichotomies may be considered as one of the most reasonable procedures. That is, our consideration in this section is summarized as follows.

Let $P_{S}$ and $P_{T}$ be the means of two binomial populations which denote the lethal (or effective) percentages of standard preparation and that of the test preparation respectively. Then a difference of the toxicity (or the effectiveness, etc.) between two preparations, $S$ and $T$, may be obtained usually by $P_{T}-P_{S}$ or by $P_{T} / P_{S}$. But the size itself of $P_{T}-P_{S}$ or $P_{T} / P_{S}$ to be used in comparing with two preparations is under the strong influence of actual sizes of the absolute values of percentages $P_{S}$ and $P_{T}$, and it is much more so in practical situation where the observations obtained by a routine assay method may suffer from some block variations (days, places of a laboratory and so on). Consequently another function of $P_{s}$ and $P_{T}$ is better sought for to be used in our present formulation.

Now let $\left(a_{i}, b_{i}\right)$ be a pair of results in $i$-th simultaneous trial $(i=1,2$, $\cdots, N)$ and let us consider only the pairs $(0,1)$ and $(1,0)$, where $a$ and $b$ are the outcomes of the observations from the assay of standard preparation and test preparation and 1 and 0 denote a death and a survival of subject, respectively. Then the probability that $(a, b)=(1,0)$ is equal to $P_{S}$ - $\left(1-P_{T}\right)$ and the probability that $(a, b)=(0,1)$ is equal to $P_{T}\left(1-P_{S}\right)$. Hence, knowing that $(a, b)$ is equal to one of the pairs $(0,1)$ and $(1,0)$, the conditional probability that it is equal to $(0,1)$ is given by $P=\left(1-P_{S}\right) P_{T} /\left\{P_{S}(1\right.$ $\left.\left.-P_{T}\right)+P_{T}\left(1-P_{s}\right)\right\}$ and the conditional probability that it is equal to $(1,0)$ is 1 $-P=P_{S}\left(1-P_{T}\right) /\left\{P_{S}\left(1-P_{T}\right)+P_{T}\left(1-P_{S}\right)\right\}$. And in order to devise a proper test for testing the hypothesis that $P_{T} \geq P_{S}$, we shall state what risks of making wrong decisions we are willing to tolerate. The toxicity of test 
preparation may be measured by a ratio (the odds) $k_{2}=P_{2} /\left(1-P_{2}\right)$ of lethals to survivals and test preparation may be regarded the more toxic, the larger the value of $k_{2}$ becomes. The relative superiority of test preparation over standard preparation can reasonably be measured by a ratio of $k_{2}$ to $k_{1}\left(=P_{1} /\left(1-P_{1}\right)\right)$, that is, $u=P_{2}\left(1-P_{1}\right) / P_{1}\left(1-P_{2}\right)$. If $u=1$, the toxicity of two preparations is equally and if $u>1$, test preparation is more toxic than another, and further, if $u<1$, standard preparation is more toxic than another.

Therefore this method may be considered immediately as a testing method concerning a difference between the means of two binomial distributions without a condition of normal approximation, Wald [1].

Now let us consider three binomial distributions and let $P_{i} \quad(i=1,2,3)$ be an unknown parameter showing the probability of a success (death, effectiveness, etc) in a single trial of the $i$-th binomial population. Our problem is to test the hypothesis $P_{2}=P_{3}$ finally on the basis of the oddsratio $u$ defined by the observations of the same number $N$ on each population, Now our rule for the procedure is formulated as follows.

(i) Let $v_{1}$ and $v_{2}$ be defined by

$$
\left\{\begin{array}{l}
v_{1}=1 / 2, \\
v_{2}=u_{2} /\left(1+u_{2}\right)=P_{2}\left(1-P_{1}\right) /\left\{P_{2}\left(1-P_{1}\right)+P_{1}\left(1-P_{2}\right)\right\} .
\end{array}\right.
$$

(ii) Let $l$ be a number of the favourable pairs between $S_{(1)}$ and $S_{(2)}$ in each sequence of $N$ and let $m$ be a number of pairs that show us the effectiveness of $S_{(2)}$ against $S_{(1)}$ in $l$ pairs. Then the number $m$ is compared with a number $k_{1}\left(\right.$ or $\left.k_{2}\right)$, where $k_{1}\left(\right.$ or $\left.k_{2}\right)$ satisfies the following relation:

$$
\sum_{i=k_{1}}^{l}\left(\begin{array}{l}
l \\
i
\end{array}\right)\left(\frac{1}{2}\right)^{l} \leq \alpha_{1} \text {, for the alternative hypothesis } P_{2}>P_{1},
$$

or

$$
\sum_{i=0}^{k_{2}}\left(\begin{array}{l}
l \\
i
\end{array}\right)\left(\frac{1}{2}\right)^{l} \leq \alpha_{1} \text {, for the alternative hypothesis } P_{2}<P_{1}
$$

(iii) Now let us introduce a test for $T_{2}$ defined in the following manner.

(a) If $m \geq k_{1}$ (or $\left.m \leq k_{2}\right)$, we define $v_{1}^{\prime}$ and $v_{2}^{\prime}$ by

$$
\left\{\begin{array}{l}
v_{1}^{\prime}=1 / 2 \\
v_{2}^{\prime}=P_{3}\left(1-P_{2}\right) /\left\{P_{3}\left(1-P_{2}\right)+P_{2}\left(1-P_{3}\right)\right\},
\end{array}\right.
$$

and the following test procedure is carried out finally.

Let $l^{\prime}$ be a number of the favourable pairs betewen $S_{(2)}$ and $T_{2}$ in each sequence of $N$ and let $m^{\prime}$ be a number of pairs that show us the effectiveness of $T_{2}$ against $S_{(2)}$ in $l^{\prime}$ pairs. Then the number $m^{\prime}$ is compared with 
a number $k_{1}^{\prime}$ (or $k_{2}^{\prime}$ ), where $k_{1}^{\prime}$ (or $k_{2}^{\prime}$ ) satisfies the following relation.

$$
\sum_{i=k_{1}^{\prime}}^{l^{\prime}}\left(\begin{array}{c}
l^{\prime} \\
i
\end{array}\right)\left(\frac{1}{2}\right)^{l \prime} \leq \alpha_{2} \text {, for the alternative hypothesis } P_{3}>P_{2} \text {, }
$$

or

$$
\sum_{i=0}^{k_{2}^{\prime}}\left(\begin{array}{c}
l^{\prime} \\
i
\end{array}\right)\left(\frac{1}{2}\right)^{\prime^{\prime}} \leq \alpha_{2}, \text { for the alternative hypothesis } P_{3}<P_{2} .
$$

(b) If $m<k_{1}$ (or $\left.m>k_{2}\right)$, we define $v_{1}^{\prime \prime}$ and $v_{2}^{\prime \prime}$ by

$$
\left\{\begin{aligned}
v_{1}^{\prime \prime}= & 1 / 2, \\
v_{2}^{\prime \prime}= & \left\{P_{3}\left(1-P_{1}\right)+P_{3}\left(1-P_{2}\right)\right\} /\left\{P_{1}\left(1-P_{3}\right)+P_{2}\left(1-P_{3}\right)+P_{3}\left(1-P_{1}\right)\right. \\
& \left.+P_{3}\left(1-P_{2}\right)\right\},
\end{aligned}\right.
$$

and the following test procedure is carried out finally.

Let $l^{\prime \prime}$ be $l^{\prime}+l_{1}$ and let $m^{\prime \prime}$ be $m^{\prime}+m_{1}$, where $l^{\prime}$ and $m^{\prime}$ are defined by (a) and let $l_{1}$ be defined by a number of the favourable pairs between $T_{2}$ and $S_{(1)}$ in each sequence of $N$, and let $m_{1}$ be a number of pairs that show us the effectiveness of $T_{2}$ against $S$ in $l_{1}$ pairs. Then the number $m^{\prime \prime}$ is compared with a number $k_{1}^{\prime \prime}$ (or $\left.k_{1}^{\prime \prime}\right)$ where $k_{1}^{\prime \prime}$ (or $k_{2}^{\prime \prime}$ ) satisfies the following relation.

$$
\sum_{i=k_{1}^{\prime \prime}}^{\prime \prime \prime}\left(\begin{array}{c}
l^{\prime \prime} \\
i
\end{array}\right)\left(\frac{1}{2}\right)^{l^{\prime \prime}} \leq \alpha_{3}, \quad\left(P_{3}=P_{2}\right)
$$

or

$$
\sum_{i=0}^{k_{2}^{\prime \prime}}\left(\begin{array}{c}
l^{\prime \prime} \\
i
\end{array}\right)\left(\frac{1}{2}\right)^{l \prime \prime} \leq \alpha_{3}, \quad\left(P_{3}<P_{2}\right)
$$

Now we assume under the consideration of numerical calculation that a correlation of $\left\{P_{1}\left(1-P_{3}\right)+P_{3}\left(1-P_{1}\right)\right\}$ and $\left\{P_{3}\left(1-P_{2}\right)+P_{2}\left(1-P_{3}\right)\right\}$ may and now shall be neglected on the basis of the fact that this correlation is approximately given by $P_{2}\left(1-P_{2}\right) / N$ for moderate large value of $N$, e.g., it becomes 0.0125 in case that $N=20$ and $P_{2}=0.5$, and 0.0045 in case that $N=20$ and $P_{2}=0.9$.

Then the power according to our test procedure is shown as follows approximately :

$$
\begin{aligned}
\sum_{i=k 1}^{l}\left(\begin{array}{l}
l \\
i
\end{array}\right) v_{2}^{i}\left(1-v_{2}\right)^{l-i} \sum_{j=k_{1^{\prime}}}^{l^{\prime}}\left(\begin{array}{l}
l^{\prime} \\
j
\end{array}\right) v_{2}^{\prime j}\left(1-v_{2}^{\prime}\right)^{l^{\prime-j}} \\
+\sum_{i=0}^{k_{1}-1}\left(\begin{array}{l}
l \\
i
\end{array}\right) v_{2}^{i}\left(1-v_{2}\right)^{l-i} \sum_{j=k_{1^{\prime \prime}}}^{l^{\prime \prime}}\left(\begin{array}{c}
l^{\prime \prime} \\
j
\end{array}\right) v_{2}^{\prime \prime j}\left(1-v_{2}^{\prime \prime}\right)^{i \prime-j} .
\end{aligned}
$$

Now in case of testing a lethal ratio for test preparation, where the hy. 
pothesis that $P_{3}=P_{2}$ is tested against an alternative hypothesis that $P_{3}>P_{2}$ and our successive procedure of this test is defined by using $k_{2}$ first and then by using $k_{1}^{\prime}$ or $k_{1}^{\prime \prime}$ secondly, the behaviour of the size of the power and the power gain are given in Fig. $5\left(\alpha_{1}=\alpha_{2}=\alpha_{3}=0.05\right)$ and in Fig. $6\left(\alpha_{1}=\right.$ $\left.0.20, \alpha_{2}=\alpha_{3}=0.05\right)$, for $N=20$ and for each $\left(P_{1}, P_{2}, P_{3}\right)$ of all the combinations of $30 \%, 50 \%$ and $70 \%$.

In these figures, we note that each number of the favourable pairs depends on a combination of each value of $\left(P_{1}, P_{2}, P_{3}\right)$ and the nominal level of 0.05 is not given on the basis of each percentagetest, but is defined on the basis of odds ratio test. From a general view of these figures, we may obtain some resonable explanations as in $\S 3$. That is to say, we may prefer to take about $20 \%$ as $\alpha_{1}$ with an intension of size control and from the view-point of the power gain. Here we need to introduce some assumptions in regard to a distance between $P_{1}$ and $P_{3}$.

$\left(B_{1}\right)$
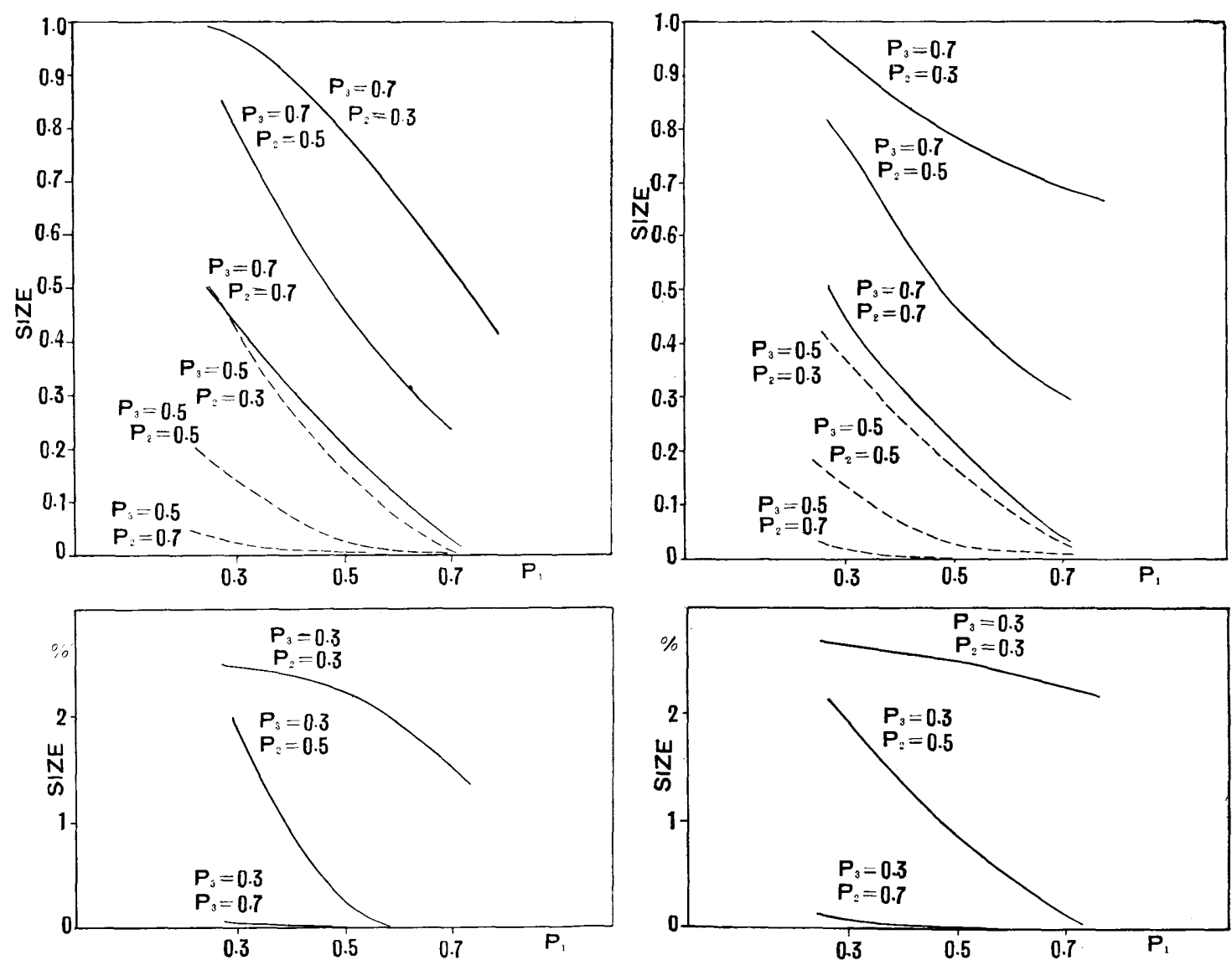

Fig. 5. (a) Size curves for $N=20, \alpha_{1}=\alpha_{2}=\alpha_{3}$ $=0.05$



Fig. 6. (a) Size curves for $N=20, \alpha_{1}=0.20$, $\alpha_{2}=\alpha_{3}=0.05$ 
$\left(\mathrm{B}_{2}\right)$
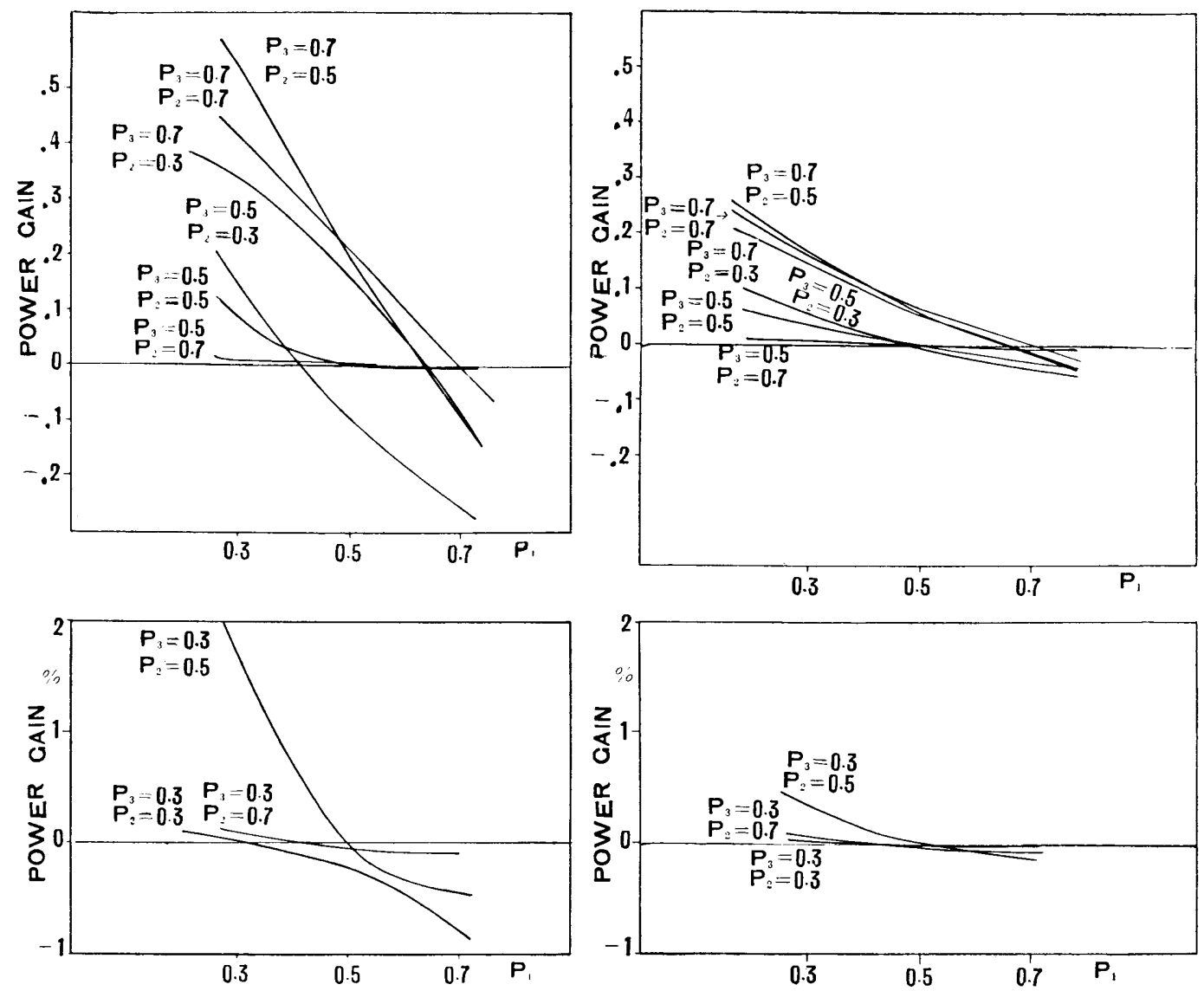

Fig. 5. (b) Power gainfor $N=20, \alpha_{1}=\alpha_{2}$ $=\alpha_{3}=0.05$

Fig. 6. (b) Power gain for $N=20, \alpha_{1}=0.20$, $\alpha_{2}=\alpha_{3}=0.05$

In conclusion, if we can assume a situation $P_{3}>P_{1}$, we may propose to take our sometimes pooling procedure taking $20 \%$ as $\alpha_{1}$, and otherwise if we can assume a situation $P_{3}<P_{1}$, a never-pool test should be recommended to be used, and when we have nothing to assume, we may propose a borderline test.

Kyushu University and Shionogi Pharmaceutical Co.

\section{References}

Bennett, B. D. [1] : Estimation of means on the basis of preliminary tests of significance, Ann. Inst. Stat. Math., Tokyo, 4 (1952), 31-43.

Bozivich, H., Bancroft, T. A. and Hartlet, H. O. [1] : Power of analysis of variance test procedures for certain incompletely specified models, I, Ann. Math. Stat., 27 (1956), 1017-1043.

Kitagawa, T. [1] : Successive process of statistical inferences, Mem. Faculty of Sci., Kyushu Univ., Ser. A, 15 (1950), 139-180.

FinNex, J. D. [1] : Statistical Methods in Biological Assay, Charles Griffin \& Co., (1952), 21-57.

WaLD, A. [1] : Sequential Analysis, John Wiley \& Sons, (1952), 106-116. 